\title{
Model Order Determination and Segmentation of Analog-Digital Converters Integral Non Linearity
}

\author{
Samer Medawar*, Peter Händel*, Niclas Björsell ${ }^{\dagger}$ and Magnus Jansson* \\ ${ }^{*}$ Signal Processing Lab \\ ${ }^{\dagger}$ Center for RF Measurememt Technology \\ ACCESS Linnaeus Center \\ SE-100 44 Stockholm,Sweden \\ University of Gävle \\ SE-801 76 Gävle, Sweden
}

Email: samer.medawar@ee.kth.se

\begin{abstract}
Analog-digital converter (ADC) integral nonlinearity (INL) modeling is investigated. The model is comprised of two entities: a low code frequency (LCF) component modeled by an $L$-order polynomial, and a static high code frequency component (HCF), modeled by $P$ linear disjoint segments centered around zero. Both model components are functions of the ADC output code $k$. A methodical way of estimating the LCF polynomial order $L$ and the set of segments (number of and their borders), is suggested. The method computes the polynomial order $L$ and the set of segments (number and borders) that minimizes the root mean square (RMS) distance between the INL data and its model. The method is applied to measured INL sequences of a 12-bit Analog Devices pipeline ADC (AD9430).
\end{abstract}

Index Terms-Analog-digital converter, integral nonlinearity, least-squares solution, polynomial model order, segmentation.

\section{INTRODUCTION}

The analog-digital converter (ADC) is not an ideal device. The integral nonlinearity (INL), a figure of merit for ADCs represents the deviation of a practical quantizer from an ideal staircase quantizer and is defined for every ADC output code $k$ [1]. For a given ADC resolution (i.e. the number of bits $N$ which leads to $2^{N}$ output codes), the INL sequence can constitute a data set of considerable size, especially if it is characterized for several input frequencies over a wide band. Thus, developing a low-complexity model that represents the INL data has been a topic of research interest, especially because such a model could potentially be used for ADC postcorrection (e.g., replacing a look-up-table (LUT) that requires the storage of a large amount of data.)

The INL data of pipeline ADCs has two major attributes, or they can alternatively be said to comprise two main components. The first is an abrupt or sharp trend occurring in consecutive intervals that is denoted in the literature as the high code frequency (HCF) component. The HCF is related to the pipeline structure and, thus, is a rather static entity. The second trend in the INL data is a smooth and continuous polynomial-shaped variation, often denoted as the low code frequency (LCF) component.

A dynamic INL model consisting of a single term was developed in [2]. A dynamic model depends on extra parameters in addition to the ADC output code $k$. The model was represented by a two-dimensional polynomial, for which the slope of the signal is added to the code dimension to represent

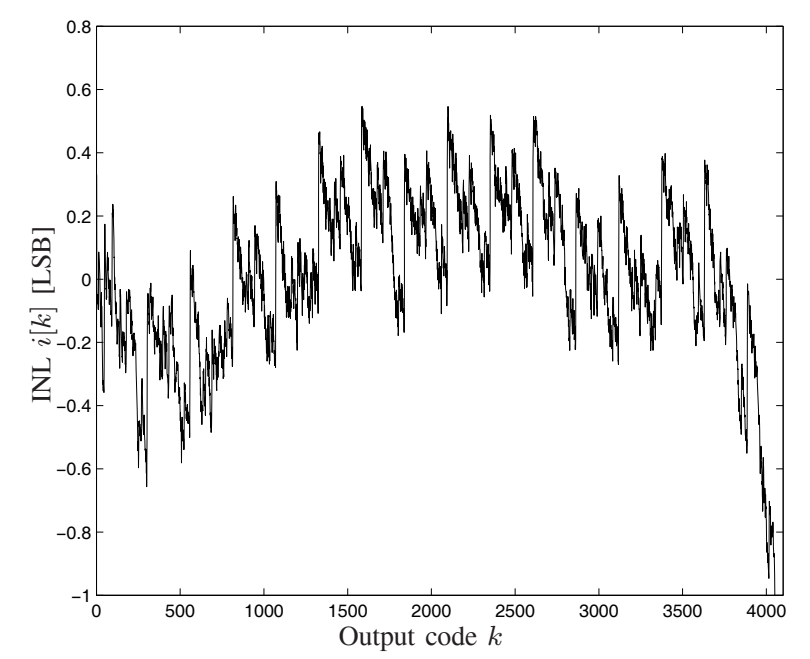

Fig. 1. The average INL for 15 sequences corresponding to test frequencies in the interval 30-90 $\mathrm{MHz}$ for a 12-bit pipelined ADC (AD9430).

the INL. Static INL models were presented in [3]-[6], where the LCF was modeled by a low order polynomial. A dynamic INL model composed of two terms was proposed in [7]. The $\mathrm{HCF}$ term was modeled by static piecewise-linear segments, whereas the LCF was determined as a dynamic polynomial that depends on the frequency of the input stimuli. The model was parameterized in [8], and the parameters were estimated using the method of least-squares. The method was applied on simulated data. In [9], modifications of the method in [8] were suggested; experimental data were also used in the evaluation. An INL model similar to [8] but with centered (around the zero axis) piecewise linear segments for the HCF model was developed in [10]. The modeling method in [10] was furthermore found to be effective in representing the INL data.

However, none of the aforementioned papers present a method to compute the LCF polynomial order $L$ as well as the number of HCF segments and their borders. In [8]-[10], an ad hoc method to determine the number of segments and their respective borders was used. Furthermore, substantial manual intervention was needed to acquire the final set of segments. 
In this paper, a methodical procedure is proposed to compute the LCF polynomial order $L$ and the number of segments and borders for the HCF. In short, a minimization criterion that determines the best fit between the INL data and the parametrized model is developed to compute the model characteristics prior to the parameters least-squares estimation. The paper is organized as follows: Sec. II describes the INL characterization, modeling, and least-squares estimation. The model order determination and segmentation process are presented in Sec. III. Sec. IV presents the results, and Sec. V concludes the work.

\section{INTEgRAL NONLINEARITY MODELING}

\section{A. The INL data}

The INL is the measure of practical ADC deviations compared to an ideal staircase quantizer [11], [12]. An ideal ADC code transition levels $T_{k}$ [Volt] can be written in terms of the ADC output code $k$ as [1]

$$
T_{k}=Q \cdot(k-1)+T_{1} \quad[\text { Volt }],
$$

where $Q$ [Volt] is the ideal width of a code bin: that is, the full-scale range of the ADC divided by the total number of codes $\left(V_{\max }-V_{\min }\right) / 2^{N}$. The quantity $T_{1}$ is the first transition level.

The ADC is not an ideal device, thus, the actual transition levels are designated by $T[k]$ [Volt]. In addition, the output of an ADC suffers certain gain and offset errors. The INL $i[k]$ is defined as the difference between $T_{k}$ given in (1) and $T[k]$, after correcting for the gain and offset [1]:

$$
i[k]=\frac{T_{k}-G \cdot T[k]-V_{\mathrm{os}}}{Q}[\mathrm{LSB}]
$$

where $G$ and $V_{\mathrm{os}}$ are the gain factor and offset term, respectively. The gain and offset can be found using the least-squares method, or, alternatively, by enforcing a null residual at the terminal codes. An average INL sequence measured for a 12bit pipelined ADC (AD9430), over a $60 \mathrm{MHz}$ band (30-90 $\mathrm{MHz}$ ) is shown in Fig. 1.

The INL data have shown some dependency on the input frequency and it is designated further on by $i[k, m]$. A dynamic INL model $i_{m, k}$ is developed in this paper, similarly to the dynamic INL data $i[m, k]$. The INL model $i_{m, k}$ consists of two entities: a static HCF that depends on the ADC output code $k$, and a dynamic LCF having an additional dependency on the input frequency $m$. The relation between the INL data $i[m, k]$ and the INL model $i_{m, k}$ reads:

$$
i[m, k]=i_{m, k}+e_{m, k}=h_{k}+l_{m, k}+e_{m, k}
$$

where $e_{m, k}$ is an error term that indicates the information the model can not represent. The quantities $h_{k}$ and $l_{m, k}$ are the HCF and LCF models that will be detailed subsequently.

The HCF is modeled by a set of $P$ linear piecewise segments centered around zero. Thus, for an output code $k$ lying in a given segment $p(p=1, \ldots, P)$, the HCF component model can be written as

$$
h_{k} \triangleq h_{k, p}=\left(k-\frac{k_{p-1}+k_{p}-1}{2}\right) \eta_{p},
$$

$k_{p-1}$ and $k_{p}-1$ are the edges of a given interval segment $p$, i.e. $k_{p-1} \leq k<k_{p}$, where the first and last ADC output codes are $k_{0}=1$ and $k_{P}=2^{N}$, respectively. The quantity $\eta_{p}$ is the segment $p$ gain or slope. The respective segment slopes are stacked in the vector

$$
\boldsymbol{\eta}=\left(\eta_{1}, \ldots, \eta_{P}\right)^{T},
$$

where $T$ denotes transpose. The output codes for the segment $p$, can be gathered in the vector

$$
\mathbf{h}_{p}=\left(\begin{array}{c}
-\frac{k_{p}-k_{p-1}-1}{2} \\
\vdots \\
\frac{k_{p}-k_{p-1}-1}{2}
\end{array}\right) .
$$

Thus, introducing the matrix $\mathbf{h}$ as

$$
\mathbf{h}=\left(\begin{array}{ccc}
\mathbf{h}_{1} & 0 & \\
0 & \ddots & 0 \\
& 0 & \mathbf{h}_{P}
\end{array}\right)
$$

allows for representation of the HCF vector model over the whole code range as $\mathbf{h} \eta$. The first and last segments $p=1$ and $p=P$, are not centered and are given, respectively, as

$$
\mathbf{h}_{1}=\left(\begin{array}{c}
0 \\
1 \\
\vdots \\
k_{1}-2
\end{array}\right) \quad \mathbf{h}_{P}=\left(\begin{array}{c}
k_{P-1}-\left(k_{P}-1\right) \\
\vdots \\
1 \\
0
\end{array}\right) .
$$

The LCF component $\ell_{m, k}$ is modeled in (3) by an $L$-th order polynomial in normalized codes $\bar{k}$, that is

$$
\bar{k}=\frac{k-2^{N-1}}{2^{N-1}-1} .
$$

Thus, for a given input frequency $f_{m}$ (where $m=1, \ldots, M$ ), the LCF model can be written in terms of the normalized code $\bar{k}$ as

$$
\ell_{m, k}=\theta_{0, m}+\theta_{1, m} \bar{k}+\cdots+\theta_{L, m} \bar{k}^{L}=\boldsymbol{\ell}_{k}^{T} \boldsymbol{\theta}_{m} .
$$

In (10), $\boldsymbol{\theta}_{m}$ is a vector (of length $L+1$ ) that contains the LCF polynomial coefficients for a given frequency $f_{m}$

$$
\boldsymbol{\theta}_{m}=\left(\theta_{0, m}, \ldots, \theta_{L, m}\right)^{T}
$$

and $\ell_{k}$ is a regressor vector of length $2^{N}-1$ that stacks the normalized code $\bar{k}$ values

$$
\ell_{k}=\left(\begin{array}{c}
1 \\
\frac{k-2^{N-1}}{2^{N-1}-1} \\
\vdots \\
\left(\frac{k-2^{N-1}}{2^{N-1}-1}\right)^{L}
\end{array}\right)
$$


A given LCF vector model is denoted as $\boldsymbol{\ell}_{\boldsymbol{m}}=\boldsymbol{\ell} \boldsymbol{\theta}_{m}$ where $\ell$ is a Vandermonde matrix:

$$
\boldsymbol{\ell}=\left(\begin{array}{cccc}
1 & -1 & \cdots & (-1)^{L} \\
\vdots & \vdots & & \vdots \\
1 & \frac{k-2^{N-1}}{2^{N-1}-1} & \cdots & \left(\frac{k-2^{N-1}}{2^{N-1}-1}\right)^{L} \\
\vdots & \vdots & & \vdots \\
1 & 1 & \cdots & 1^{L}
\end{array}\right)
$$

Prior to determining the order $L$ and the set of $P$ segments, the INL model parameters estimation is first presented, for a given $L$ and $P$. For multiple frequency $m$ characterizations the INL-model-data relation can be expressed in a set of linear equations as in [13]. The INL model parameters are obtained as in [13]; the HCF segments slopes are

$$
\widehat{\boldsymbol{\eta}}=\left(\mathbf{h}^{T} \boldsymbol{\pi}_{\perp} \mathbf{h}\right)^{-1} \mathbf{h}^{T} \boldsymbol{\pi}_{\perp} \overline{\mathbf{i}},
$$

where $\boldsymbol{\pi}_{\perp}=\mathbf{I}-\ell\left(\ell^{T} \ell\right)^{-1} \ell^{T}$ is a projection matrix, and $\overline{\mathbf{i}}=$ $\frac{1}{M} \sum_{m=1}^{M} \mathbf{i}[m]$ is the arithmetic average (with respect to the frequency) of the INL data.

The LCF polynomials coefficients solution is obtained as

$$
\widehat{\boldsymbol{\theta}}_{m}=\left(\boldsymbol{\ell}^{T} \boldsymbol{\ell}\right)^{-1} \boldsymbol{\ell}^{T}(\mathbf{i}[m]-\mathbf{h} \widehat{\boldsymbol{\eta}})=\boldsymbol{\ell}_{\mathbf{I}}^{\dagger}(\mathbf{i}[m]-\mathbf{h} \widehat{\boldsymbol{\eta}}) .
$$

The LCF model is refined into a static and dynamic model, where the static or average LCF polynomial coefficients are obtained as

$$
\overline{\boldsymbol{\theta}}=\frac{1}{M} \sum_{m=1}^{M} \widehat{\boldsymbol{\theta}}_{m}=\boldsymbol{\ell}_{\mathbf{I}}^{\dagger}(\overline{\mathbf{i}}-\mathbf{h} \widehat{\boldsymbol{\eta}})
$$

and the dynamic LCF model components are given by (for $m=1, \ldots, M)$

$$
\tilde{\boldsymbol{\theta}}_{m}=\widehat{\boldsymbol{\theta}}_{m}-\overline{\boldsymbol{\theta}} .
$$

The complete estimated INL model for a given input frequency $m$ now reads as

$$
\widehat{\mathbf{i}}_{m}=\mathbf{h} \widehat{\boldsymbol{\eta}}+\boldsymbol{\ell} \widehat{\boldsymbol{\theta}}_{m}=\underbrace{\mathbf{h} \widehat{\boldsymbol{\eta}}+\boldsymbol{\ell \boldsymbol { \theta }}}_{\text {static part }}+\underbrace{\ell \tilde{\boldsymbol{\theta}}_{m}}_{\text {dynamic part }} .
$$

\section{HCF SEgmentation ANd LCF MOdel ORder $L$ DETERMINATION}

\section{A. A Cost Function for the Segmentation}

The HCF is a static quantity that can be modeled by a set of centered segments. The number of segments $P$ as well as their borders, denoted by $k_{1}, \ldots, k_{P-1}$ remain to be determined. Because the HCF segments are frequency-independent, a cost function $F(\mathbf{h})$ to be minimized, is the norm of the error vector between the average INL data average $\overline{\mathbf{i}}$ and the static INL model $\ell \overline{\boldsymbol{\theta}}+\mathbf{h} \widehat{\boldsymbol{\eta}}$, or

$$
F(\mathbf{h})=\|\overline{\mathbf{i}}-\ell \overline{\boldsymbol{\theta}}(\mathbf{h})-\mathbf{h} \widehat{\boldsymbol{\eta}}(\mathbf{h})\| .
$$

The sub-vectors $\mathbf{h}_{p}$ constituting the block diagonal matrix $\mathbf{h}$ are formulated in terms of the $p$-th segment that each is of the same size of a sub-vector $\mathbf{h}_{p}$. Thus, optimizing the number of HCF segments and their widths results in optimizing the structure (the number of columns) $\mathbf{h}$ and size of the subvectors $\mathbf{h}_{p}$. The length of each column in $\mathbf{h}$ is fixed to $2^{N}-1$. $\widehat{\boldsymbol{\eta}}(\mathbf{h})$ and the $\overline{\boldsymbol{\theta}}(\mathbf{h})$ in (19) are given by (14) and (16), but the $\mathbf{h}$ dependency is made explicit here because it is the optimization variable.

Replacing $\overline{\boldsymbol{\theta}}$ of (16) in (19) gives

$$
F(\mathbf{h})=\left\|\overline{\mathbf{i}}-\ell \ell_{\mathbf{I}}^{\dagger} \overline{\mathbf{i}}+\ell \ell_{\mathbf{I}}^{\dagger} \mathbf{h} \widehat{\boldsymbol{\eta}}(\mathbf{h})-\mathbf{h} \widehat{\boldsymbol{\eta}}(\mathbf{h})\right\| .
$$

Recalling $\pi_{\perp}$ defined in the previous section, (20) is rewritten to

$$
F(\mathbf{h})=\left\|\boldsymbol{\pi}_{\perp}(\overline{\mathbf{i}}-\mathbf{h} \widehat{\boldsymbol{\eta}}(\mathbf{h}))\right\|
$$

$\pi_{\perp}$ is a projection matrix aimed at deducting a polynomial of order $L$ from given data set. For HCF, $\mathbf{h} \widehat{\boldsymbol{\eta}}(\mathbf{h}) \approx \boldsymbol{\pi}_{\perp} \mathbf{h} \widehat{\boldsymbol{\eta}}(\mathbf{h})$ makes a fair approximation and will be used instead in the sequel. In contrast, the average INL data $\overline{\mathbf{i}}$ have polynomial attributes (the static LCF model).

The projected set

$$
\tilde{\mathbf{i}}=\boldsymbol{\pi}_{\perp} \overline{\mathbf{i}}=\overline{\mathbf{i}}-\ell \ell_{\mathbf{I}}^{\dagger} \overline{\mathbf{i}},
$$

makes a good approximation of the HCF data. Inserting the zero-centered sequence $\tilde{\mathbf{i}}$ of (22) and the HCF solution of (14) into (21) leads to

$$
F(\mathbf{h})=\left\|\tilde{\mathbf{i}}-\mathbf{h}\left(\mathbf{h}^{T} \boldsymbol{\pi}_{\perp} \mathbf{h}\right)^{-1} \mathbf{h}^{T} \boldsymbol{\pi}_{\perp} \overline{\mathbf{i}}\right\| .
$$

$\mathbf{h}\left(\mathbf{h}^{T} \boldsymbol{\pi}_{\perp} \mathbf{h}\right)^{-1} \mathbf{h}^{T} \boldsymbol{\pi}_{\perp} \overline{\mathbf{i}}$ is the data sequence of a weighted leastsquares solution. The weighting matrix $\boldsymbol{\pi}_{\perp}$ aims to rescind the polynomial attributes in the mean INL data prior to the HCF segments fit.

The cost function of (23) can be simplified by replacing the mean INL data $\overline{\mathbf{i}}$ by the centered HCF data $\tilde{\mathbf{i}}$ and $\boldsymbol{\pi}_{\perp}$ by the identity matrix. Doing this, the estimated HCF parameters or segment slopes are approximated by the least squares solution of the centered INL-data $\tilde{\mathbf{i}}$ as

$$
\widehat{\boldsymbol{\eta}}(\mathbf{h}) \approx\left(\mathbf{h}^{T} \mathbf{h}\right)^{-1} \mathbf{h}^{T} \tilde{\mathbf{i}}=\mathbf{h}_{\mathbf{I}}^{\dagger} \tilde{\mathbf{i}}
$$

Summing up, the resulting entity to minimize is

$$
F^{\prime}(\mathbf{h})=\left\|\tilde{\mathbf{i}}-\mathbf{h}\left(\mathbf{h}^{T} \mathbf{h}\right)^{-1} \mathbf{h}^{T} \tilde{\mathbf{i}}\right\| .
$$

The cost function (25) is used in the sequel to tune the segment edges $k_{1}, \ldots, k_{P-1}$.

\section{B. LCF Polynomial Order L}

The cost function (25) is written in terms of the HCF structure matrix $\mathbf{h}$ and the zero-centered HCF data $\tilde{\mathbf{i}}$ obtained by the projection $\tilde{\mathbf{i}}=\boldsymbol{\pi}_{\perp} \overline{\mathbf{i}}$, which phases out the polynomial form inherent to the static INL data up to a specified polynomial order $L$. Thus, a given LCF polynomial order $L$ must be large enough to be able to capture the polynomial attributes of the INL data, in order to generate a residual sequence (22) that mimics the HCF structure. However, if the polynomial order $L$ is further increased, the LCF polynomial tends to grasp the HCF structure as well. Accordingly, the order $L$ must be selected in a trade-off so that the estimated polynomial depicts the smooth behavior of the INL data while retaining the HCF modeling to the segmented model. Suppose a polynomial 


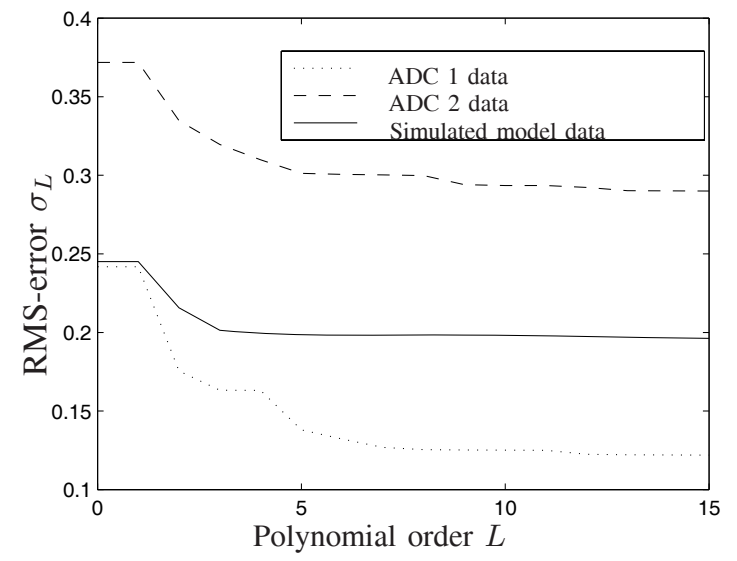

Fig. 2. Root-mean-square error of polynomial fit versus LCF polynomial order $L$ [13].

of order $L_{0}$ characterizes completely the LCF data; then, applying the projection matrix $\pi_{\perp}$ to the data results in the zero vector if $L$ that determines $\pi_{\perp}$ fulfils $L \geq L_{0}$. By performing such a polynomial fit on the average INL data $\overline{\mathbf{i}}$, the polynomial (as its order $L$ is increased) gradually depicts the INL data and their schemes vary accordingly. The rootmean-square (RMS) of the residual is an analytical measure of the LCF data and its $L$-order polynomial fit

$$
\sigma_{L}=\sqrt{\frac{\|\tilde{\mathbf{i}} \mid\|^{2}}{2^{N}-1}} .
$$

The RMS value $\sigma_{L}$ is plotted in Fig. 2 as a function of the order $L$. The method was applied on simulated data provided by the manufacturer and experimental data from two ADC samples of the same type (ADC1 and ADC2, respectively). The diagram in Fig. 2 shows that the magnitude of the residual $\mathrm{HCF}$ sequence begins to stabilize after the fifth order for the measured data. Thus, we can deduce that a polynomial order $L$ larger than 5 is needed to trade between LCF and HCF modeling. To maintain a safety margin, $L$ is chosen to be $L=7$ throughout this work.

\section{Determining the Number of HCF Segments $P$}

The cost function of (25) should be minimized with respect to the number of segments and their respective borders. In other words, one can consider

$$
\left(\widehat{P}, \widehat{k}_{1}, \ldots, \widehat{k}_{P-1}\right)=\arg \min F^{\prime}(\mathbf{h}),
$$

where $F^{\prime}(\mathbf{h})$ was defined in (25). We propose an approach to determine the number of segments $P$ by making use of the HCF data $\tilde{\mathbf{i}}$ structure prior to optimizing the respective widths $\left(\left\{k_{p}\right\}_{p=1}^{P}\right)$ of the segments. The HCF data $\tilde{\mathbf{i}}$ are quite noisy, and it is evident from Fig. 1 that only adds to the HCF $\tilde{\mathbf{i}}$ sequence a polynomial of order $L$. To find the segment borders, the data can be de-noised so that its large scale structure becomes obvious. A low pass filter is used to smooth the HCF data of (22). The HCF data of one of the two ADCs and its

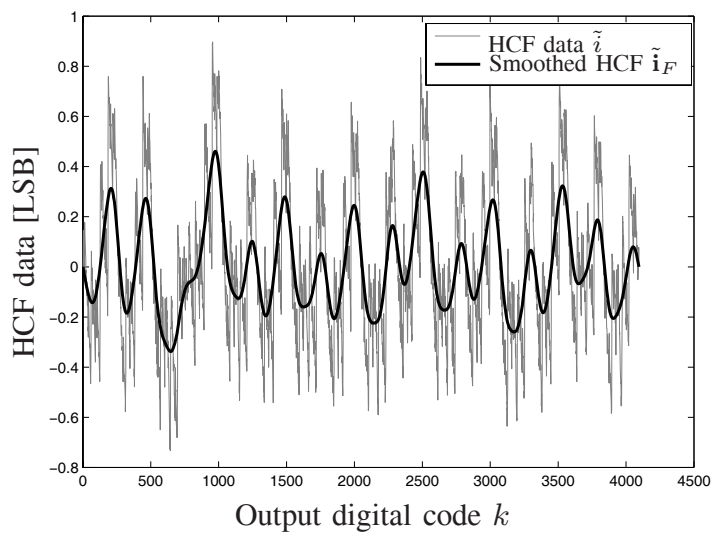

Fig. 3. Filtered version $\tilde{\mathbf{i}}_{F}$ of the calculated HCF data $\tilde{\mathbf{i}}$, obtained from the measured average INL $\overline{\mathbf{i}}$, as a function of the output digital code $k$ [13].

TABLE I

ALGORITHM FOR SELECTING THE HCF NUMBER OF SEGMENTS $P$ AND THE INITIAL GUESS ABOUT THEIR BORDERS $\left\{k_{p}\right\}$. NEGATIVE SLOPES [13].

1) Set the first segment border at $k_{0}=1$.
Select the code bin $k^{\prime}$ representing the $\tilde{\mathbf{i}}_{F}$ minimum between
the first bin $k=1$ and first zero-crossing that yields
$k_{1}=k^{\prime}+1$.
Select the code bin representing the $\tilde{\mathbf{i}}_{F}$ minimal value
between the second and third zero-crossing as the
border of the third segment.
Continue by choosing the segments borders as the minimum
$\tilde{\mathbf{i}}_{F}$ value between each pair of consecutive, disjoint zero-crossings
until the last code bin $k=2^{N}-1$ is reached.
The number of segments $P$ is obtained by adding 1 to the
number of zero-crossings.

smoothed sequence are shown in Fig. 3. The data are filtered in the forward and reverse directions to preserve the phase information.

The filtered sequence $\tilde{\mathbf{i}}_{F}$ is the basis to compute the number of segments $P$ and a reasonable initial guess about their borders $\left\{k_{p}\right\}_{p=1}^{P}$. The algorithm to calculate $P$ from $\tilde{\mathbf{i}}_{F}$ is summarized in Tab. I, where it is assumed that a negative slope is common for all the centered segments. Having obtained the segments number $P$, the corresponding associated segments borders $k_{1}, \ldots, k_{P-1}$ are then fed into a numerical optimizer to be fine-tuned by the minimization (25).

Once the proper $L, P$, and the optimized set of $k_{p}$ 's are found, the LCF and HCF components of the INL data are obtained by solving the least-squares problem studied in Sec. II.

\section{Implication of the Segmentation Method on Synthetic Data}

Exemplary synthetic HCF data based on a set of segments with a total length of 1000 were generated with variable noise powers. The segments had equal lengths and negative slopes.

The number of segments was computed by the method presented above and was found to be the same as in the original data set for all cases with different noise powers. The optimization procedure for the segment borders was then 


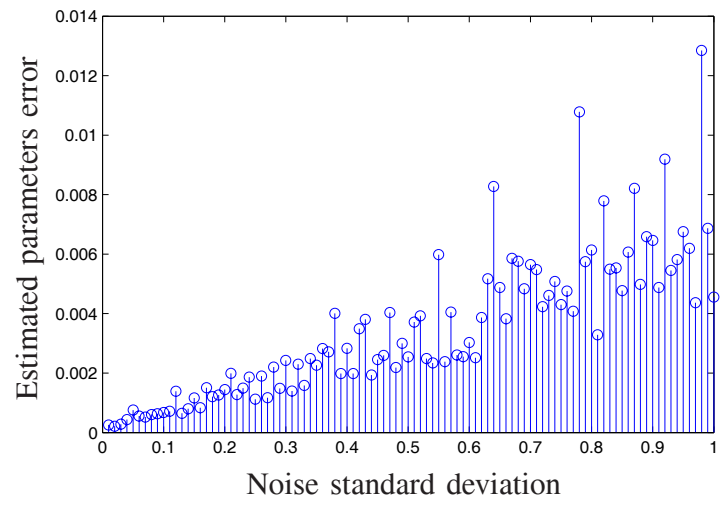

Fig. 4. Estimated model parameter error with respect to the original synthetic parameters plotted against the standard noise deviation
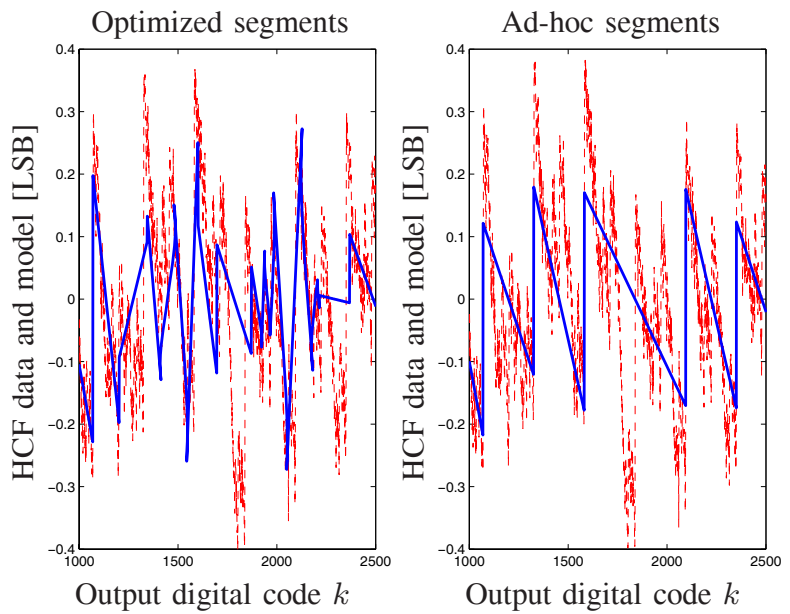

Fig. 5. Measured INL data (dashed lines), and HCF segments (solid lines) before and after the optimization process.

performed. The estimated HCF model parameters error from the original noiseless ones is plotted against the standard deviation of the noise in Fig. 4. The estimation error grows linearly with the standard deviation of the noise until a certain stage, where it begins to diverge for some isolated values.

\section{EXPERIMENTAL INVESTIGATION}

\section{A. ADC Under Test}

The ADC under test was a commercial 12-bit pipeline Analog Devices AD9430 having a maximum sampling frequency of $210 \mathrm{MHz}$. More information about the ADC and the test bed in general can be found in [14].

\section{B. Results}

The outcome of this method on the INL model structure is most obvious for the HCF component and is illustrated in Fig. 5, where a part of the HCF data and model is emphasized to show the improvements obtained using this method. The model segments achieve a much better fit with a fewer number of segments (simpler model).

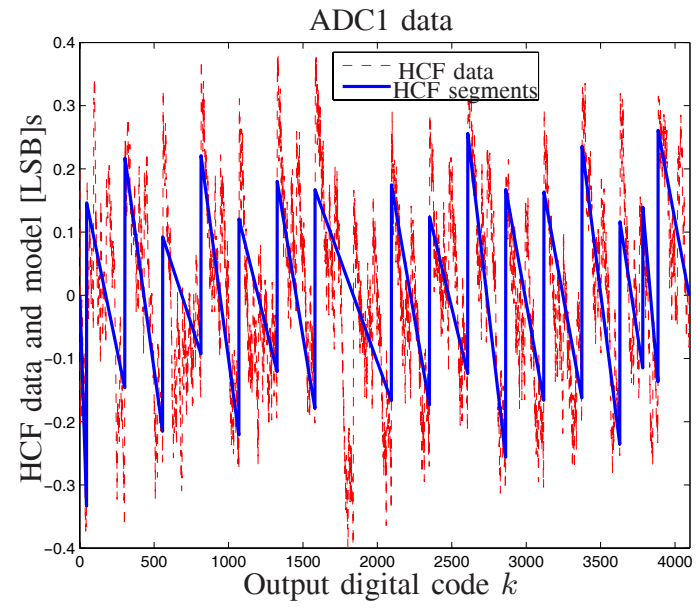

Fig. 6. HCF data and centered segments model in LSBs as a function of the digital output code $k$.

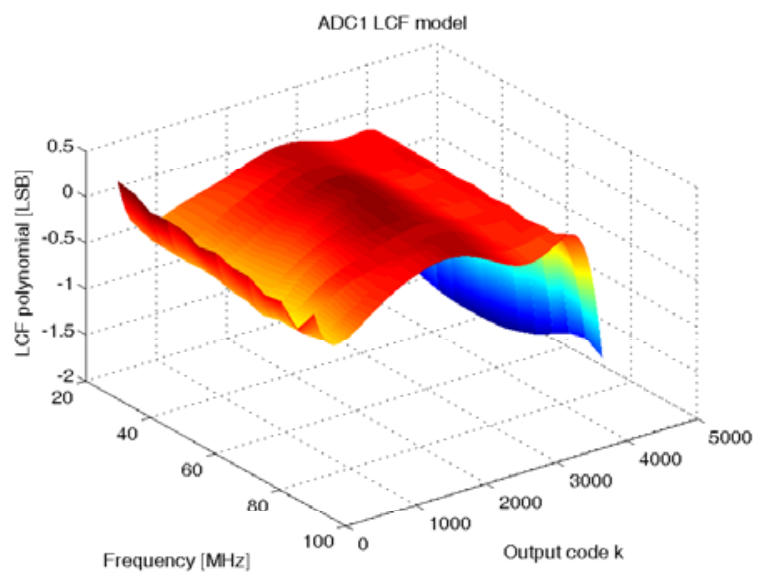

Fig. 7. LCF polynomial model in LSBs as a function of the digital output code $k$, over the frequency range.

The ADC model was described by $P=16$ segments and an $L=7$ :th-order polynomial. The HCF data and model $\mathbf{h} \widehat{\eta}$ are presented in Fig. 6, as a function of the ADC output code $k$.

Fig. 7 shows the dynamic LCF model polynomials $\boldsymbol{\ell} \widehat{\boldsymbol{\theta}}_{m}$ of (15) as a function of the code $k$, with respect to the input frequency, i.e., $m=1, \ldots, M$.

\section{CONCLUSION}

ADC INL has been characterized and modeled in this paper. The model presented consists of two components: the LCF and HCF. The LCF is modeled by a low order polynomial, and the HCF by a set of static piecewise linear segments centered around zero. The LCF polynomial order and the number and borders of HCF segments have been determined using a systematic approach. Once these characteristic parameters are obtained, the model component coefficients (i.e., LCF polynomial coefficients and HCF segment slopes) can be estimated by the least-squares method. The model was found 
to mimic the INL data well, and is suitable for post-correction.

\section{REFERENCES}

[1] IEEE Standard for Terminology and Test Methods for Analog-to-Digital Converters, IEEE Standard 1241-2000.

[2] P. Arpaia, P. Daponte and L. Michaeli, "A dynamic error model for integrating analog-to-digital converters," Measurement 25, pp. 255-264, June 1999.

[3] L. Michaeli, P. Michalko and J. Saliga, "Identification of ADC error model by testing of the chosen code bins," 12:th IMEKO TC4 International Symposium, September 25-27, 2002, Zagreb, Croatia.

[4] A. Cruz Serra, F. Alegria, L. Michaeli, P. Michalko, J. Saliga, "Fast ADC testing by repetitive histogram analysis," IEEE Instrumentation and Measurement Technology Conference, April 24-27, pp. 1633-1638, 2006.

[5] L. Michaeli, P. Michalko, J. Saliga, "Unified ADC nonlinearity error model for SAR ADC," Measurement, Vol. 41, Issue 2, pp. 198-204, February 2008.

[6] A.C. Serra, M.F. da Silva, P.M. Ramos, R.C. Martins, L. Michaeli and J. Saliga, "Combined spectral and histogram analysis for fast ADC testing," IEEE Transactions on Instrumentation and Measurement, Vol. 54, No. 4, pp. 1617-1623, August 2005.

[7] N. Björsell and P. Händel, "Dynamic behavior models of analog to digital converters aimed for post-correction in wideband applications," 11th Workshop on ADC Modelling and Testing, September 17-22, 2006, Rio de Janerio, Brazil.

[8] P. Händel, N. Björsell and M. Jansson, "Model based dynamic characterization of analog-digital-converters at radio frequency- Invited paper," 2007 International Conference on Signal Processing and its Applications, 12-15 February 2007, Sharjah, UAE.

[9] S. Medawar, N. Björsell, P. Händel and M. Jansson, "Dynamic characterization of analog-digital-converters non-linearities, "Mosharaka International Conference on Wireless Communications and Mobile Computing, Amman, Jordan 6-8 September 2007.

[10] S. Medawar, P. Handel, N. Bjorsell, M. Jansson, "ADC Characterization by Dynamic Integral non-linearity, "13th Workshop on ADC Modeling and Testing, Florence, Italy, pages 1-6, 22-24 September 2008.

[11] S. Rapuno, P. Daponte, E. Balestrieri, L. de Vito, S. J. Tilden, S. Max and J. Blair, "ADC parameters and Characteristics - part 6 in a series of tutorials in instrumentation and measurement," IEEE Instrumentation \& Measurement Magazine, Vol. 8, No. 5, pp. 44-54, December 2005.

[12] T.E. Linnenbrink, J. Blair, S. Rapuno, P. Daponte, E. Balestrieri, L. de Vito, S. Max and S. J. Tilden, "ADC testing - part 7 in a series of tutorials in instrumentation and measurement," IEEE Instrumentation \& Measurement Magazine, Vol. 9, No. 2, pp. 39-49, April 2006.

[13] S. Medawar, P. Händel, N. Björsell and M. Jansson, "Input Dependent Integral Nonlinearity Modeling for Pipelined Analog-Digital Converters," IEEE Transactions on Instrumentation and Measurements, in press December 2009.

[14] N.Björsell, O. Andersen, and P. Händel,"High dynamic test-bed for characterization of analogue-to-digital converters up to 500MSPS," 10th Workshop on ADC modeling and testing, Gdynia, Poland, pp. 601-604, 2005. 